\title{
Review of: "Chronic kidney disease and pregnancy outcomes"
}

\author{
Mariko Miyazaki ${ }^{1}$ \\ 1 Tohoku University
}

Potential competing interests: The author(s) declared that no potential competing interests exist.

This article was the pregnancy outcome in CKD, it was interesting for me, especially, the difference of low birth weight risk was shown between lupus nephritis and IgA nephropathy in figure 2.

We were also investigating the outcome of the pregnancy in 89 CKD women in our hospital. ( Kumakura S, et al. Kidney function, blood pressure and proteinuria were associated with pregnancy outcomes of pregnant women with chronic kidney disease: a single-center, retrospective study in the Asian population. Clin Exp Nephrol. 2020 Jun;24(6):547-556. doi: 10.1007/s10157-020-01865-0. Epub 2020 Mar 11. PMID:

32162117.)

In our study, blood pressure had great impact on pregnancy outcome. I am interested the impact of blood pressure in this study population.

Moreover, since the ratio of the cesarean section was $64 \%$, this may be large in a researcher's country. How was the comparison with/without CKD?

Our research member think that the simple concept of CKD defined by proteinuria and creatinine level are convenient for maternal care, however, we recognized again the cause of CKD is also important to evaluate the risk.

In order to improve the pregnancy outcome in CKD patients, the obstetrics and nephrology departments should share their medical issue and analysis.

Minor: For nephrologists, $3 \mathrm{~g}$ or more proteinuria was great differences than $1-3 \mathrm{~g}$ or $<1.0 \mathrm{~g}$ when managing pregnant women. Did authors try to draw the x-axis using logarithm in figure 1 data ? 